\title{
A Convenient, $\mathrm{TiCl}_{4} / \mathrm{SnCl}_{4}$-Mediated Synthesis of $\mathrm{N}$-Phenyl or $\boldsymbol{N}$-Aryl Benzamidines and $\mathbf{N}$-Phenylpicolinamidines
}

\author{
Umesh D. Patil and Pramod P. Mahulikar \\ School of Chemical Sciences, North Maharashtra University, Jalgaon 425 001, India \\ Correspondence should be addressed to Pramod P. Mahulikar, mahulikarpp@rediffmail.com
}

Received 24 April 2012; Accepted 19 June 2012

Academic Editors: J. Mlochowski, D. Sémeril, and B. Zacharie

Copyright (C) 2012 U. D. Patil and P. P. Mahulikar. This is an open access article distributed under the Creative Commons Attribution License, which permits unrestricted use, distribution, and reproduction in any medium, provided the original work is properly cited.

A new, $\mathrm{TiCl}_{4}$ - or $\mathrm{SnCl}_{4}$-mediated, solvent-free method was developed for the synthesis of $\mathrm{N}$-Aryl benzamidines and $\mathrm{N}$ phenylpicolinamidines, in moderate-to-good yield, using suitable amines and nitriles as starting materials.

\section{Introduction}

The amidine nucleus is found in a wide variety of biologically active molecules [1]. N-Aryl amidine exhibits activity against Mycobacterium tuberculosis, and $\mathrm{N}$-alkylfuramidine shows antiprotozoan and antimicrobial activities [2]. Similarly various amidines derived from 4-amidino2-(2-pyridyl)quinazoline [3] and 1-amino-3-(2-pyridyl)isoquinoline [3], guanidine [4], diguanidino [5], reversed diamidino 2,5-diarylfuran [5], benzimidazole [6], pyridine [7], exhibit antimycoplasmal, antimalarial, antimicrobial, antibacterial, anti-inflammatory activities. An extensive number of monoamidines have been evaluated for their utility in blocking various stages of the thrombin cascade, and numerous highly potent molecules have been reported [8]

Amidines were used as important synthon in organic synthesis in the preparation of various heterocyclic compounds, such as pyridine $[7,9]$, pyrimidines $[9,10]$, imidazoles [11], pyrazolopyrimidine [12], iminopyrimidine [13], imidazopyridine and pyrimidinopyridine [14], purine [15], benzimidazole [16], pyrimidines [17], triazaphenalene [18], triazine [19], tetraazole [19], thiadiazine [20], oxazolotriazole [21], diazirine [22], triazolopyridine [23], azetidinone [24], and pyrrole, and also used as complexing agent [25].

Several synthetic strategies have been developed for the synthesis of amidines, in which the nucleophilic addition of amine to nitrile is the most popular. Generally, nitriles were activated to the intermediate salt in the presence of $\mathrm{EtOH} / \mathrm{HCl}$ [26] or $\mathrm{NH}_{4} \mathrm{Cl} / \mathrm{MeOH}$ [27] under anhydrous condition and then reacted with amine to get amidine. While for unreactive nitriles, Lewis acid or other condensing agents were used such as anhydrous $\mathrm{AlCl}_{3}, \mathrm{ZnCl}_{2}$ [28], $\mathrm{CuCl}$ [29], Ln (III) salts [30], $\mathrm{CaCl}_{2}$ [31], $\mathrm{Al}\left(\mathrm{CH}_{3}\right)_{3}$ [32], $\mathrm{SmI}_{2}$ [33], Ytterbium amide [34], $\mathrm{MeSO}_{3} \mathrm{H}$ [20], and anhyd. $\mathrm{SnCl}_{4}$ [21]. Amides can be converted to imidoyl chloride using $\mathrm{PCl}_{5}[35,36]$, which can then react with primary or secondary amine to yield amidine. In addition, amide can be O-alkylated with triethyloxonium fluoroborate at ambient temperature to yield the corresponding imidic ester fluoroborate, which then reacts with amine to yield the targeted amidine [3]. Iron pentacarbonyl was employed to the conversion of amidoximes into amidines via reductive cleavage of the $\mathrm{N}=\mathrm{O}$ bond [37]. Sometimes, strong bases like LiHMDS, NaHMDS, LDA, BuLi, $\mathrm{NaOMe} \mathrm{[38],} \mathrm{and} \mathrm{NaH}$ [20] were used as condensing agent. Similarly, Dains F. B. has shown that amidine was prepared from symmetrical diaryl and dialkyl urea and acid chloride [39, 40].

In 1998, Zhou and Zhang published the results on such a subject that amidines were successfully prepared from nitriles and nitrocompounds in the presence of $\mathrm{TiCl}_{4} / \mathrm{Sm}$ in THF. They also reported that under same reaction conditions amidine formation was not observed by treatment of nitriles with amines [41]. Thus, it was of interest to study the reactions of nitrile and amine using $\mathrm{TiCl}_{4}$ and $\mathrm{SnCl}_{4}$. 
TABLE 1: $\mathrm{SnCl}_{4} / \mathrm{TiCl}_{4}$ catalysed coupling of substituted anilines with benzonitrile.

\begin{tabular}{lcccc}
\hline Entry no. & Amines & \multicolumn{2}{c}{ Amidines $^{\text {a }}$ (isolated yield \%) } & M.P. ${ }^{\circ} \mathrm{C}(\mathrm{Lit})$. \\
\hline 3a & Aniline & by $\mathrm{TiCl}_{4}$ & 71 & $114-116(116)$ \\
$3 \mathbf{b}$ & 75 & 67 & $108-110$ \\
$3 \mathbf{c}$ & 2-Cl-Aniline & 65 & 63 & $86-88$ \\
\hline
\end{tabular}

all products were characterised by IR, NMR, and mass spectral data and in comparison with authentic samples.

TABLE 2: $\mathrm{SnCl}_{4} / \mathrm{TiCl}_{4}$ catalysed coupling of 2-aminopyridine with substituted benzonitriles.

\begin{tabular}{lcccccc}
\hline Entry no. & & Amines & & Nitriles & \multicolumn{2}{c}{ Amidines $^{\text {a }}$ (isolated yield \%) } \\
by bnCl $_{4}$
\end{tabular}

all products were characterised by IR, NMR, and mass spectral data and in comparison with authentic samples.

TABLE 3: $\mathrm{SnCl}_{4} / \mathrm{TiCl}_{4}$ catalysed coupling of substituted anilines with 2-cyanopyridine.

\begin{tabular}{|c|c|c|c|c|}
\hline \multirow{2}{*}{ Entry no. } & \multirow{2}{*}{$\begin{array}{c}\text { Amines } \\
\mathrm{R}\end{array}$} & \multicolumn{2}{|c|}{ Amidines $^{\mathrm{a}}$ (isolated yield \%) } & \multirow{2}{*}{ M.P. ${ }^{\circ} \mathrm{C}$ (Lit.) } \\
\hline & & by $\mathrm{TiCl}_{4}$ & by $\mathrm{SnCl}_{4}$ & \\
\hline $3 \mathbf{i}$ & $\mathrm{H}$ & 79 & 68 & 78-80 HCl Salt \\
\hline $3 \mathbf{j}$ & $2-\mathrm{CH}_{3}$ & 59 & 64 & $68-70(68-69)$ \\
\hline $3 k$ & $4-\mathrm{CH}_{3}$ & 64 & 61 & $54(52-53)$ \\
\hline 31 & $4-\mathrm{F}$ & 78 & 69 & $72(75-76)$ \\
\hline $3 m$ & $4-\mathrm{Cl}$ & 66 & 60 & $80-82(80-82)$ \\
\hline $3 n$ & $4-\mathrm{Br}$ & 81 & 65 & $84-86(85-86)$ \\
\hline 30 & $3,4-\mathrm{Cl}$ & 76 & 69 & $112(112-113)$ \\
\hline
\end{tabular}

all products were characterised by IR, NMR, and mass spectral data and in comparison with authentic samples.

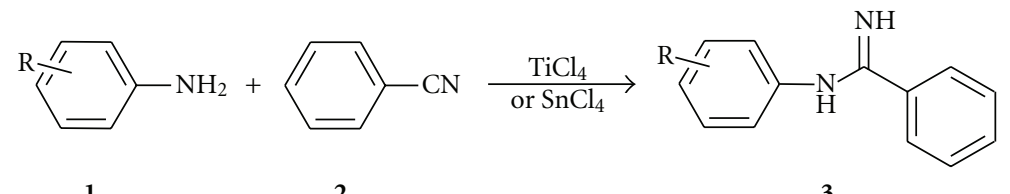

Scheme 1

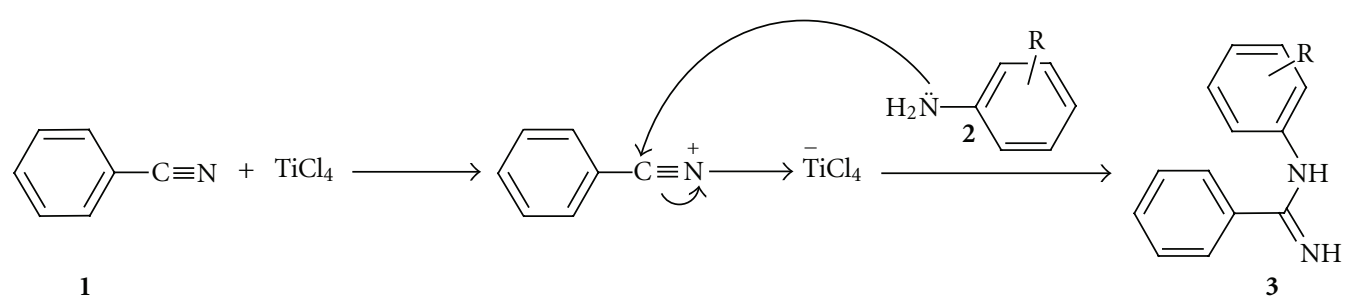

Scheme 2: Mechanism.

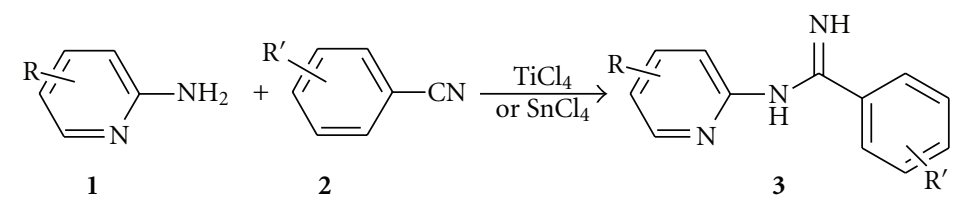

SCHEME 3 


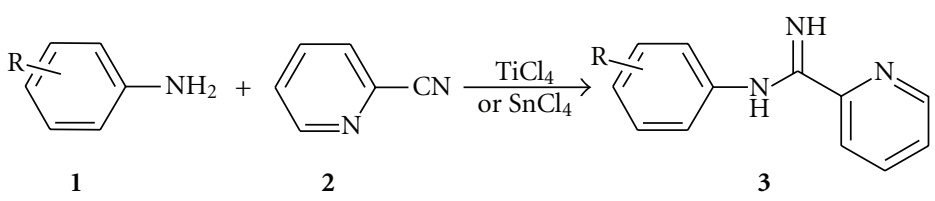

Scheme 4

\section{Results and Discussion}

We would like to demonstrate in the present work that amidine could be prepared by coupling nitrile with amine in presence of $\mathrm{TiCl}_{4}$ as well as $\mathrm{SnCl}_{4}$ using neat condition in absence of samarium. At the beginning we studied the synthesis of amidine (Scheme 1, Table 1, and Entry 1) using benzonitrile and aniline as model substrates. In a typical experiment aniline $(0.01 \mathrm{~mol})$ and benzonitrile $(0.01 \mathrm{~mol})$ were heated at $100-110^{\circ} \mathrm{C}$ with $\mathrm{TiCl}_{4}$ or $\mathrm{SnCl}_{4}(0.012 \mathrm{~mol})$ for $3-4 \mathrm{hr}$ to complete the reaction. The obtained black reaction mixture was then neutralised with $\mathrm{NaOH}$ solution and extracted with dichloromethane. Product was isolated simply by evaporation of the solvent at reduced pressure. The crude product was recrystallized from hexane. The obtained product was characterised by IR, NMR, and mass spectroscopy data and compared with authentic sample. Furthermore, the reaction was carried out for several substituted aryl amines and nitriles (Table 1) under the same conditions. It is distinct that both $\mathrm{TiCl}_{4}$ and $\mathrm{SnCl}_{4}$ were found to have a potential utility for the synthesis of amidine with good-tomoderate yields under mentioned reaction conditions.

The mechanism we propose for the reaction is similar to the one reported for amidine using $\mathrm{AlCl}_{3}$ [42] and is outlined in Scheme 2: at first the complex between nitrile and $\mathrm{TiCl}_{4}$ was formed followed by nucleophilic addition of amine (2) on the more electrophilic carbon of nitriles to yield the amidine (3).

With these results in hand, we tested the scope and limitations of this process; we examined the coupling reaction of various substituted benzonitriles with heteroaromatic amine, that is, 2-aminopyridine, (Scheme 3 and Table 2) by performing the reaction with the well-established reaction conditions.

Similarly, to test the scope and limitations of this process; we examined the coupling reaction of various substituted anilines with heteroaromatic nitrile, that is, 2-cyanopyridine, (Scheme 4 and Table 3 ) by performing the reaction with the well-established reaction conditions.

\section{Conclusion}

In the summary, we have developed a solvent-free method of amidine formation from nitrile and amine using $\mathrm{TiCl}_{4}$ or $\mathrm{SnCl}_{4}$ in absence of expensive metal-like samarium. The reaction proceeded at $100-110^{\circ} \mathrm{C}$ and was completed within 3-4 hrs. In conclusion the reaction was extremely simple to carry out, and the obtained yield of amidine was good to moderate. On the basis of yield, we may conclude that $\mathrm{TiCl}_{4}$ is preferable catalyst over $\mathrm{SnCl}_{4}$.

\section{Experimental}

Melting points were determined by open capillary tube method and are uncorrected. Progress of the reaction was monitored by TLC (visualization was effected by exposure to UV light). Commercial reagents were used without purification for synthesis. Mass spectra were recorded on Thermo Finnigan (model- LCQ Advantage MAX) mass spectrometer. The IR spectra were recorded with $\mathrm{KBr}$ pellets on Perkin-Elmer Spectrum One Spectrometer. ${ }^{1} \mathrm{H}$ NMR spectra were recorded in $\mathrm{CDCl}_{3}$ on a Bruker $300 \mathrm{DRX}$ Avance instrument at $300 \mathrm{MHz}$.

4.1. Preparation of Amidines, 3a-o. Benzonitrile (1.03 g, $0.01 \mathrm{~mol}$ ) was taken in a dry round bottom flask and to this was added a 2-aminopyridine $(0.94 \mathrm{~g}, 0.01 \mathrm{~mol})$. The flask was heated, after fitting a dry condenser along with a guard tube, in an oil bath at a temperature range of $80-90^{\circ} \mathrm{C}$ with stirring. After $30 \mathrm{~min} \mathrm{TiCl}_{4}(1.3 \mathrm{~mL}, 0.012 \mathrm{~mol})$ or $\mathrm{SnCl}_{4}$ $(1.4 \mathrm{~mL}, 0.012 \mathrm{~mol})$ was added to the flask. After addition, temperature was increased to $100-110^{\circ} \mathrm{C}$, and contents of the flask were heated for 3-4 hrs. The mixture was cooled to room temperature, and the solid, thus, formed was dissolved in hot water and made alkaline with $10 \% \mathrm{NaOH}$. This solution was extracted with a $\mathrm{CH}_{2} \mathrm{Cl}_{2}(3 \times 100 \mathrm{~mL})$. Then organic layer was decolourized with activated charcoal and dried over anhydrous $\mathrm{Na}_{2} \mathrm{SO}_{4}$. After evaporating the solvent under reduced pressure, crude amidine was obtained. This crude product was recrystallized from hexane to get pure amidine.

$N$-Phenylbenzamidine (3a, $\left.\mathrm{C}_{13} \mathrm{H}_{12} \mathrm{~N}_{2}\right)$. ${ }^{1} \mathrm{H} \mathrm{NMR}\left(\mathrm{CDCl}_{3}, \delta\right.$ ppm): 4.84 (br s, 2H, NH, C=NH), 6.96-6.99 (d, J=8.1 Hz, 2H, ArH), 7.03-7.08 (t, J=7.5 Hz 1H, ArH), 7.32-7.37 (t, $\mathrm{J}=7.8 \mathrm{~Hz}, 2 \mathrm{H}, \mathrm{ArH}), 7.42-7.49$ (m, 3H, ArH), 7.85 (s, 2H, $\mathrm{ArH})$; IR (KBr) v $\left(\mathrm{cm}^{-1}\right): 3340,2853,1618,1574,1459,1377$, 1153, 722; MS (ESI, $70 \mathrm{eV}) \mathrm{m} / z$ (\%): 198 (13), 197 (100) [M $+\mathrm{H}]^{+}$.

$\mathrm{N}$-(2-Chlorophenyl)benzamidine $\quad\left(3 \boldsymbol{b}, \quad \mathrm{C}_{13} \mathrm{H}_{11} \mathrm{ClN}_{2}\right) .{ }^{1} \mathrm{H}$ $\mathrm{NMR}\left(\mathrm{CDCl}_{3}, \delta \mathrm{ppm}\right): 4.78$ (br s, $\left.2 \mathrm{H}, \mathrm{NH}, \mathrm{C}=\mathrm{NH}\right)$, 6.97-7.02 (m, 2H, ArH), 7.19-7.22 (t, J=7.2 Hz,1H, ArH), 7.39-7.51 (m, 4H, ArH), 7.86 (br s, 2H, ArH); IR (KBr) v $\left(\mathrm{cm}^{-1}\right): 3345,2853,1618,1574,1459,1377,1153,722 ; \mathrm{MS}$ (ESI, $70 \mathrm{eV}) \mathrm{m} / z(\%): 233(37), 231(100)[\mathrm{M}+\mathrm{H}]^{+}$.

$\mathrm{N}$-(4-Fluorophenyl)benzamidine $\left(3 c, \mathrm{C}_{13} \mathrm{H}_{11} \mathrm{FN}_{2}\right)$. ${ }^{1} \mathrm{H} \mathrm{NMR}$ $\left(\mathrm{CDCl}_{3}, \delta \mathrm{ppm}\right): 4.86$ (br s, $\left.2 \mathrm{H}, 2 \mathrm{NH}\right), 6.90-6.94(\mathrm{~m}$, $2 \mathrm{H}, \mathrm{ArH}), 7.01-7.07(\mathrm{t}, \mathrm{J}=8.3 \mathrm{~Hz}, 2 \mathrm{H}, \mathrm{ArH}), 7.41-7.46(\mathrm{t}$, $\mathrm{J}=7.3 \mathrm{~Hz}, 3 \mathrm{H}, \mathrm{ArH}), 7.83-7.86(\mathrm{~d}, \mathrm{~J}=6.3 \mathrm{~Hz}, 2 \mathrm{H}, \mathrm{ArH})$; IR 
$(\mathrm{KBr}) \nu\left(\mathrm{cm}^{-1}\right): 3348,2854,1625,1590,1459,1377,1152$, 777, 722; MS (ESI, $70 \mathrm{eV}) \mathrm{m} / z$ (\%): 216 (17), 215 (100) $[\mathrm{M}+$ $\mathrm{H}]^{+}, 198(13)$.

$\mathrm{N}$-(Pyridin-2-yl)benzamidine $\left(3 \boldsymbol{d}, \mathrm{C}_{12} \mathrm{H}_{11} \mathrm{~N}_{3}\right) .{ }^{1} \mathrm{H} \quad \mathrm{NMR}$ $\left(\mathrm{CDCl}_{3}, \delta \mathrm{ppm}\right): 2.03$ (bs, 2H, 2NH), 6.90-6.95 (m, 1H, ArH), 7.25-7.29 (d, J=9 Hz, 1H, ArH), 7.40-7.48 (m, 3H, ArH), 7.61-7.67 (m, 1H, ArH), 7.89-7.92 (m, 2H, ArH), 8.31-8.34 (m, 1H, ArH); IR (KBr) v $\left(\mathrm{cm}^{-1}\right)$ : 3351, 2854, $1625,1590,1459,1377,1152,777,722$; MS (ESI, $70 \mathrm{eV}) \mathrm{m} / z$ (\%): $198(100)[\mathrm{M}+\mathrm{H}]^{+}$.

3-Chloro-N-(pyridin-2-yl)benzamidine (3e, $\left.\mathrm{C}_{12} \mathrm{H}_{10} \mathrm{ClN}_{3}\right)$. ${ }^{1} \mathrm{H} \mathrm{NMR}\left(\mathrm{CDCl}_{3}, \delta \mathrm{ppm}\right): 2.03$ (bs, 2H, 2NH), 6.92-6.96 $(\mathrm{m}, 1 \mathrm{H}, \mathrm{ArH}), 7.25-7.45(\mathrm{~m}, 3 \mathrm{H}, \mathrm{ArH}), 7.62-7.68(\mathrm{~m}$, $1 \mathrm{H}, \mathrm{ArH}), 7.72-7.73$ (d, J=1.5 Hz, 1H, ArH), 7.92-7.93 (t, $\mathrm{J}=1.8 \mathrm{~Hz}, 1 \mathrm{H}, \mathrm{ArH}), 8.32-8.34(\mathrm{~m}, 1 \mathrm{H}, \operatorname{ArH})$; IR $(\mathrm{KBr}) v$ $\left(\mathrm{cm}^{-1}\right)$ : 3341, 2854, 1625, 1590, 1459, 1377, 1152, 777, 722; MS (ESI, $70 \mathrm{eV}) \mathrm{m} / z(\%): 231(100)[\mathrm{M}+\mathrm{H}]^{+}$.

4-Chloro-N-(pyridin-2-yl)benzamidine (3f, $\left.\mathrm{C}_{12} \mathrm{H}_{10} \mathrm{ClN}_{3}\right)$. ${ }^{1} \mathrm{H} \mathrm{NMR}\left(\mathrm{CDCl}_{3}, \delta \mathrm{ppm}\right): 1.81$ (br s, $\left.2 \mathrm{H}, 2 \mathrm{NH}\right), 6.93-6.97$ (m, 1H, ArH), 7.25-7.27 (t, J=8.1 Hz,1H, ArH), 7.41-7.43 (d, J=6.3 Hz, 2H, ArH), 7.63-7.69 (m, 1H, ArH), 7.85-7.89 (d, J=6.9 Hz, 2H, ArH), 8.33-8.35 (m, 1H, ArH); IR (KBr) v $\left(\mathrm{cm}^{-1}\right)$ : 3344, 2853, 1618, 1594, 1462, 1377, 1125, 831, 782, 722, 538; MS (ESI, $70 \mathrm{eV}) \mathrm{m} / z(\%): 231(100)[\mathrm{M}+\mathrm{H}]^{+}$.

4-Bromo-N-(pyridin-2-yl)benzamidine (3g, $\left.\mathrm{C}_{12} \mathrm{H}_{10} \mathrm{BrN}_{3}\right)$. ${ }^{1} \mathrm{H} \mathrm{NMR}\left(\mathrm{CDCl}_{3}, \delta \mathrm{ppm}\right): 1.78$ (br s, $2 \mathrm{H}, 2 \mathrm{NH}, \mathrm{C}=\mathrm{NH}$ ), 6.92-6.97 (m, 1H, ArH), 7.24-7.27 (t, J=3.9 Hz,1H, ArH), 7.55-7.60 (m, 2H, ArH), 7.63-7.69 (m, 1H, ArH), 7.72-7.81 (m, 2H, ArH), 8.32-8.35 (m, 1H, ArH); IR (KBr) v $\left(\mathrm{cm}^{-1}\right)$ : 3349, 2854, 1640, 1577, 1530, 1459, 1377, 1321, 1300, 1260, $1151,1119,1096,1006,818,769,722,541$; MS (ESI, $70 \mathrm{eV})$ $m / z(\%): 278(100), 276(100)[\mathrm{M}+\mathrm{H}]^{+}$.

$\mathrm{N}$-(5-Bromopyridin-2-yl)benzamidine (3h, $\left.\mathrm{C}_{12} \mathrm{H}_{10} \mathrm{BrN}_{3}\right) .{ }^{1} \mathrm{H}$ $\mathrm{NMR}\left(\mathrm{CDCl}_{3}, \delta \mathrm{ppm}\right): 1.68$ (br s, 2H, NH), 7.16-7.19 (d, 1H, ArH), 7.42-7.49 (m, 3H, ArH), 7.71-7.75 (m, 1H, ArH), 7.88-7.91 (t, J=6 Hz, 2H, ArH), 8.37-8.38 (d, J=2.4 Hz, 1H, $\mathrm{ArH})$; IR (KBr) $v\left(\mathrm{~cm}^{-1}\right): 3350,2853,1618,1574,1459,1377$, 1153, 722; MS (ESI, $70 \mathrm{eV}) \mathrm{m} / z$ (\%): 278 (94), 276 (100) [M $+\mathrm{H}]^{+}, 260(90), 259(90)$.

N-Phenylpicolinamidine (3i, $\left.\mathrm{C}_{12} \mathrm{H}_{11} \mathrm{~N}_{3}\right) .{ }^{1} \mathrm{H} \mathrm{NMR}\left(\mathrm{CDCl}_{3}, \delta\right.$ ppm): 5.91 (br s, $2 \mathrm{H}, \mathrm{NH}, \mathrm{C}=\mathrm{NH}), 6.88-7.26$ (m, 4H, ArH), 7.31-7.37 (m, 2H, ArH), 7.78-7.81 (m, 1H, ArH), 8.41-8.45 (m, 1H, ArH), 8.52-8.59 (m, 1H, ArH); IR (KBr) v $\left(\mathrm{cm}^{-1}\right)$ : 3346, 2853, 1618, 1574, 1459, 1377, 1153, 722; MS (ESI, $70 \mathrm{eV}) \mathrm{m} / z(\%): 198(100)[\mathrm{M}+\mathrm{H}]^{+}, 181(45)$.

N-o-tolylpicolinamidine (3j, $\left.\mathrm{C}_{13} \mathrm{H}_{13} \mathrm{~N}_{3}\right) .{ }^{1} \mathrm{H} \mathrm{NMR}\left(\mathrm{CDCl}_{3}, \delta\right.$ ppm): 2.18 (s, 3H, $\mathrm{ArCH}_{3}$ ), 5.72 (br s, $2 \mathrm{H}, \mathrm{NH}, \mathrm{C}=\mathrm{NH}$ ), 6.89-7.02 (m, 2H, ArH), 7.16-7.24 (q, J=7.8 Hz, 1H, ArH), $7.30-7.33$ (dd, J=8.4 Hz,1H, ArH), 7.36-7.41 (m, 1H, ArH), 7.77-7.84 (m, 1H, ArH), 8.36-8.48 (m, 1H, ArH), 8.55-8.57 $(\mathrm{m}, 1 \mathrm{H}, \mathrm{ArH})$; IR $(\mathrm{KBr}) \nu\left(\mathrm{cm}^{-1}\right): 3345,1694,1613,1463$, 1377, 1118, 721; MS (ESI, $70 \mathrm{eV}) \mathrm{m} / z$ (\%): 212 (100) $[\mathrm{M}+$ $\mathrm{H}]^{+}, 195(37)$.

$N$-p-tolylpicolinamidine $\left(3 \boldsymbol{k}, \mathrm{C}_{13} \mathrm{H}_{13} \mathrm{~N}_{3}\right) .{ }^{1} \mathrm{H} \mathrm{NMR}\left(\mathrm{CDCl}_{3}\right.$, $\delta$ ppm): 2.18 (s, 3H, $\mathrm{ArCH}_{3}$ ), 5.58 (br s, $2 \mathrm{H}, \mathrm{NH}, \mathrm{C}=\mathrm{NH}$ ), 6.90-7.01 (m, 3H, ArH), 7.18-7.26 (m, 2H, ArH), 7.36-7.40 (m, 1H, ArH), 7.56 (m, 1H, ArH), 7.77-7.84 (m, 1H, ArH); IR $(\mathrm{KBr}) \nu\left(\mathrm{cm}^{-1}\right): 3339,2854,1694,1613,1463,1377,1118$, 721; MS (ESI, $70 \mathrm{eV}) \mathrm{m} / z(\%): 212(100)[\mathrm{M}+\mathrm{H}]^{+}, 195$ (37).

$\mathrm{N}$-(4-Fluorophenyl)picolinamidine $\quad\left(3 \mathrm{l}, \quad \mathrm{C}_{12} \mathrm{H}_{10} \mathrm{FN}_{3}\right) .{ }^{1} \mathrm{H}$ $\mathrm{NMR}\left(\mathrm{CDCl}_{3}, \delta\right.$ ppm): 1.76 (br s, $\left.1 \mathrm{H}, \mathrm{NH}\right), 5.81$ (br s, $1 \mathrm{H}$, $\mathrm{NH}), 6.88-7.02(\mathrm{~m}, 4 \mathrm{H}, \mathrm{ArH}), 7.32-7.35(\mathrm{~m}, 1 \mathrm{H}, \mathrm{ArH})$, 7.73-7.78 (m, 1H, ArH), 8.32-8.34 (d, J=6 Hz, 1H, ArH), 8.49-8.50 (d, J=6 Hz, 1H, ArH); IR $(\mathrm{KBr}) \nu\left(\mathrm{cm}^{-1}\right)$ : 3378, 2854, 1625, 1590, 1459,1377, 1152, 777, 722; MS (ESI, $70 \mathrm{eV})$ $m / z(\%): 216(100)[\mathrm{M}+\mathrm{H}]^{+}, 199(25)$.

$\mathrm{N}$-(4-Chlorophenyl)picolinamidine $\left(3 \mathrm{~m}, \mathrm{C}_{12} \mathrm{H}_{10} \mathrm{ClN}_{3}\right) .{ }^{1} \mathrm{H}$ $\mathrm{NMR}\left(\mathrm{CDCl}_{3}, \delta \mathrm{ppm}\right): 5.86$ (br s, $\left.2 \mathrm{H}, \mathrm{NH}, \mathrm{C}=\mathrm{NH}\right), 6.84-$ $6.88(\mathrm{~m}, 2 \mathrm{H}, \mathrm{ArH}), 7.25-7.42(\mathrm{~m}, 3 \mathrm{H}, \mathrm{ArH}), 7.73-7.82(\mathrm{t}$, $\mathrm{J}=5.7 \mathrm{~Hz}, 1 \mathrm{H}, \mathrm{ArH}), 8.29-8.40$ (m, 1H, ArH), 8.51-8.59 (m, $1 \mathrm{H}, \mathrm{ArH})$; IR (KBr) v $\left(\mathrm{cm}^{-1}\right): 3345,2853,1618,1574,1459$, 1377, 1153, 722; MS (ESI, $70 \mathrm{eV}) \mathrm{m} / z$ (\%): 232 (100) $[\mathrm{M}+$ $\mathrm{H}]^{+}, 215(33)$.

$\mathrm{N}$-(4-Bromophenyl)picolinamidine (3n, $\left.\mathrm{C}_{12} \mathrm{H}_{10} \mathrm{BrN}_{3}\right) .{ }^{1} \mathrm{H}$ $\mathrm{NMR}\left(\mathrm{CDCl}_{3}, \delta \mathrm{ppm}\right): 5.85$ (br s, $2 \mathrm{H}, \mathrm{NH}, \mathrm{C}=\mathrm{NH}$ ), 6.88-6.91 (d, J=8.7 Hz, 2H, ArH), 7.38-7.50 (m, 3H, ArH), 7.79-7.84 (m, 1H, ArH), 8.36-8.39 (d, J=8.1 Hz, 1H, ArH), 8.56-8.58 (m, 1H, ArH); IR (KBr) v $\left(\mathrm{cm}^{-1}\right)$ : 3376, 2854, $1640,1577,1530,1459,1377,1321,1300,1260,1151,1119$, 1096, 1006, 818, 769, 722, 541; MS (ESI, $70 \mathrm{eV}) \mathrm{m} / z$ (\%): 278 (100), $276(100)[\mathrm{M}+\mathrm{H}]^{+}, 260(20), 259(20)$.

$\mathrm{N}$-(3,4-Dichlorophenyl)picolinamidine (3o, $\left.\mathrm{C}_{12} \mathrm{H}_{9} \mathrm{Cl}_{2} \mathrm{~N}_{3}\right)$. ${ }^{1} \mathrm{H} \mathrm{NMR}\left(\mathrm{CDCl}_{3}, \delta \mathrm{ppm}\right): 5.97$ (br s, $\left.2 \mathrm{H}, 2 \mathrm{NH}\right), 6.84-6.88$ (m, 1H, ArH), 7.12-7.17 (t, 1H, ArH), 7.39-7.43 (t, J=3.9 Hz, $2 \mathrm{H}, \mathrm{ArH}), 7.79-7.87(\mathrm{t}, \mathrm{J}=9.6 \mathrm{~Hz}, 1 \mathrm{H}, \mathrm{ArH}), 8.33-8.36(\mathrm{~d}$, $\mathrm{J}=7.8 \mathrm{~Hz}, 1 \mathrm{H}, \mathrm{ArH}), 8.56-8.58(\mathrm{~d}, \mathrm{~J}=4.5 \mathrm{~Hz}, 1 \mathrm{H}, \mathrm{ArH})$; IR $(\mathrm{KBr}) \nu\left(\mathrm{cm}^{-1}\right): 3345,1694,1613,1463,1377,1118,721 ; \mathrm{MS}$ (ESI, $70 \mathrm{eV}) \mathrm{m} / z(\%): 267(100), 266(65)[\mathrm{M}+\mathrm{H}]^{+}$.

\section{References}

[1] S. Patai and Z. Rappoport, The Chemistry of Amidines and Imidates, vol. 27, John Wiley \& Sons, New York, NY, USA, 1991.

[2] S. M. Rahmathullah, R. R. Tidwell, S. K. Jones, J. E. Hall, and D. W. Boykin, "Carbamate prodrugs of $N$-alkylfuramidines," European Journal of Medicinal Chemistry, vol. 43, no. 1, pp. 174-177, 2008.

[3] M. A. H. de Zwart, H. van der Goot, and H. Timmerman, "Synthesis and copper-dependent antimycoplasmal activity of 1-amino-3-(2-pyridyl)isoquinoline derivatives. 2. Amidines," Journal of Medicinal Chemistry, vol. 32, no. 2, pp. 487-493, 1989. 
[4] M. Calas, M. Ouattara, G. Piquet et al., "Potent antimalarial activity of 2-aminopyridinium salts, amidines, and guanidines," Journal of Medicinal Chemistry, vol. 50, no. 25, pp. 6307-6315, 2007.

[5] C. E. Stephens, F. Tanious, S. Kim et al., "Diguanidino and "reversed" diamidino 2,5-diarylfurans as antimicrobial agents," Journal of Medicinal Chemistry, vol. 44, no. 11, pp. 1741-1748, 2001.

[6] H. Göker, S. Özden, S. Yildiz, and D. W. Boykin, "Synthesis and potent antibacterial activity against MRSA of some novel 1,2-disubstituted- $1 H$-benzimidazole- $N$-alkylated-5-carboxamidines," European Journal of Medicinal Chemistry, vol. 40, no. 10, pp. 1062-1069, 2005.

[7] S. V. Bhosale, U. D. Patil, M. B. Kalyankar, S. V. Nalage, V. S. Patil, and K. R. Desale, "Facile synthesis of 2,6-diaryl4-secondary aminonicotinonitriles and highly substituted unsymmetrical 2,2'-bipyridines," Journal of Heterocyclic Chemistry, vol. 47, no. 3, pp. 691-696, 2010.

[8] R. Frédérick, L. Pochet, C. Charlier, and B. Masereel, "Modulators of the coagulation cascade: focus and recent advances in inhibitors of tissue factor, factor VIIa and their complex," Current Medicinal Chemistry, vol. 12, no. 4, pp. 397-417, 2005.

[9] R. Pratap, B. Kumar, and V. J. Ram, "An efficient substituent dependent synthesis of congested pyridines and pyrimidines," Tetrahedron, vol. 63, no. 41, pp. 10309-10319, 2007.

[10] N. Agarwal, P. Srivastava, S. K. Raghuwanshi et al., "Chloropyrimidines as a new class of antimicrobial agents," Bioorganic and Medicinal Chemistry, vol. 10, no. 4, pp. 869-874, 2002.

[11] J. F. Cheng, M. Chen, B. Liu, Z. Hou, T. Arrhenius, and A. M. Nadzan, "Design and synthesis of heterocyclic malonyl-CoA decarboxylase inhibitors," Bioorganic and Medicinal Chemistry Letters, vol. 16, no. 3, pp. 695-700, 2006.

[12] N. D. Adams, S. J. Schmidt, S. D. Knight, and D. Dhanak, "A novel synthesis of substituted $4 H$-pyrazolo[3,4-d]pyrimidin4-ones," Tetrahedron Letters, vol. 48, no. 23, pp. 3983-3986, 2007.

[13] J. A. McCauley, C. R. Theberge, and N. J. Liverton, "Chemoselective reactions of amidines: selective formation of iminopyrimidine regioisomers," Organic Letters, vol. 2, no. 21, pp. 3389-3391, 2000.

[14] M. W. Cartwright, G. Sandford, J. Bousbaa et al., "Imidazopyridine and pyrimidinopyridine systems from perfluorinated pyridine derivatives," Tetrahedron, vol. 63, no. 30, pp. 7027 7035, 2007.

[15] B. G. Szczepankiewicz, J. J. Rohde, and R. Kurukulasuriya, "Synthesis of purines and other fused imidazoles from acyclic amidines and guanidines," Organic Letters, vol. 7, no. 9, pp. 1833-1835, 2005.

[16] V. J. Grenda, R. E. Jones, G. Gal, and M. Sletzinger, "Novel preparation of benzimidazoles from $N$-arylamidines. New synthesis of thiabendazole," Journal of Organic Chemistry, vol. 30, no. 1, pp. 259-261, 1965.

[17] R. Pratap, Farahanullah, R. Raghunandan, P. R. Maulik, and V. J. Ram, "Substituent directed regioselective synthesis of 2-oxonicotonic acids and methyl nicotinates," Tetrahedron Letters, vol. 48, no. 28, pp. 4939-4942, 2007.

[18] R. Pratap, A. D. Roy, S. P. Kushwaha, A. Goel, R. Roy, and V. J. Ram, "Guanidine and amidine mediated synthesis of bridgehead triazaphenalenes, pyrimidines and pyridines through domino reactions," Tetrahedron Letters, vol. 48, no. 33, pp. 5845-5849, 2007.

[19] J. J. Shie and J. M. Fang, "Microwave-assisted one-pot tandem reactions for direct conversion of primary alcohols and aldehydes to triazines and tetrazoles in aqueous media,"
Journal of Organic Chemistry, vol. 72, no. 8, pp. 3141-3144, 2007.

[20] J. Zienkiewicz, P. Kaszynski, and V. G. Young, "Fusedring thiadiazines: preparation and crystallographic characterization of 3-phenyl derivative of benzo-, pyridio[2,3-e]-, pyrazino[2,3-e]-, and tetrafluorobenzo-[1,2,4]thiadiazines," Journal of Organic Chemistry, vol. 69, no. 7, pp. 2551-2561, 2004.

[21] T. Sambaiah and K. K. Reddy, "Synthesis of 2-Aryl[1,2,4] triazolo[5,1-b]benzoxazoles by oxidative cyclization of $N$ (benzoxazol-2-yl)benzamidines," Synthesis, no. 5, pp. 422424, 1990.

[22] W. H. Graham, "The halogenation of amidines. I. Synthesis of 3-halo- and other negatively substituted diazirines," Journal of the American Chemical Society, vol. 87, no. 19, pp. 4396-4397, 1965.

[23] K. T. Potts, H. R. Burton, and J. Bhattacharyya, "1,2,4Triazoles. XIII. Derivatives of the s-triazolo[1,5-a]pyridine ring system," Journal of Organic Chemistry, vol. 31, no. 1, pp. 260-265, 1966.

[24] A. K. Bose and I. Kugajevsky, "Studies on lactams-VII. A new synthesis of $\beta$-amino- $\beta$-lactams," Tetrahedron, vol. 23 , no. 2 , pp. 957-963, 1967.

[25] R. K. Mahajan and P. Dhawan, "Adsorptive stripping voltammetric determination of nickel(II) using N-2-pyridylbenzamidine as a complexing reagent," Indian Journal of Chemistry A, vol. 41, no. 5, pp. 981-984, 2002.

[26] R. Roger and D. G. Neilson, "The chemistry of imidates," Chemical Reviews, vol. 61, no. 2, pp. 179-211, 1961.

[27] K. Dabak, "Synthesis and protection of some amidines," Turkish Journal of Chemistry, vol. 26, no. 4, pp. 547-550, 2002.

[28] J. D. Bower and G. R. Ramage, "Heterocyclic systems related to pyrrocoline. Part II. The preparation of polyazaindenes by dehydrogenative cyclisations," Journal of the Chemical Society (Resumed), pp. 4506-4510, 1957.

[29] G. Rousselet, P. Capdevielle, and M. Maumy, "Copper(I)induced addition of amines to unactivated nitriles: the first general one-step synthesis of alkyl amidines," Tetrahedron Letters, vol. 34, no. 40, pp. 6395-6398, 1993.

[30] J. H. Forsberg, V. T. Spaziano, T. M. Balasubramanian et al., "Use of lanthanide(III) ions as catalysts for the reactions of amines with nitriles," Journal of Organic Chemistry, vol. 52, no. 6, pp. 1017-1021, 1987.

[31] M. Meder, C. H. Galka, and L. H. Gade, "Bis(2-pyridylimino)isoindole $(B P I)$ ligands with novel linker units: synthesis and characterization of their palladium and platinum complexes," Monatshefte fur Chemie, vol. 136, no. 10, pp. 1693-1706, 2005.

[32] B. Asproni, A. Pau, M. Bitti et al., "Synthesis and pharmacological evaluation of 1-[(1,2-diphenyl- $1 H$-4-imidazolyl)methyl]-4-phenylpiperazines with clozapine-like mixed activities at dopamine $\mathrm{D}_{2}$, serotonin, and $\mathrm{GABA}_{\mathrm{A}}$ receptors," Journal of Medicinal Chemistry, vol. 45, no. 21, pp. 4655-4668, 2002.

[33] F. Xu, J. Sun, and Q. Shen, "Samarium diiodide promoted synthesis of N, $\mathrm{N}^{\prime}$-disubstituted amidines," Tetrahedron Letters, vol. 43, no. 10, pp. 1867-1869, 2002.

[34] J. Wang, F. Xu, T. Cai, and Q. Shen, "Addition of amines to nitriles catalyzed by ytterbium amides: an efficient onestep synthesis of monosubstituted $\mathrm{N}$-arylamidines," Organic Letters, vol. 10, no. 3, pp. 445-448, 2008.

[35] M. W. Partridge and A. Smith, "Cyclic amidines. Part XXIV. Cyclisation of $N$-allyl- $N$ '-arylacetamidines to imidazolines, 
dihydroquinazolines, and dihydrobenzodiazepines," Journal of the Chemical Society, Perkin Transactions 1, pp. 453-456, 1973.

[36] A. J. Hill and J. V. Johnston, "Amidines derived from ethylenediamine. I. Diamidines," Journal of the American Chemical Society, vol. 76, no. 3, pp. 920-922, 1954.

[37] A. Dondoni and G. Barbaro, "Synthetic reactions using transition metal complexes. Conversion of amide oximes into amidines by pentacarbonyliron and evidence for imine intermediates in the deoximation of ketoximes," Journal of the Chemical Society, Chemical Communications, no. 18, pp. 761762, 1975.

[38] I. K. Khanna, Y. Yu, R. M. Huff et al., "Selective cyclooxygenase-2 inhibitors: heteroaryl modified 1,2-diarylimidazoles are potent, orally active antiinflammatory agents," Journal of Medicinal Chemistry, vol. 43, no. 16, pp. 3168-3185, 2000.

[39] F. B. Dains, "On the action of certain acid reagents on the substituted ureas," Journal of the American Chemical Society, vol. 22, no. 4, pp. 181-198, 1900.

[40] F. B. Dains, R. C. Roberts, and R. Q. Brewster, "On the action of certain acid reagents on the substituted ureas and thiazole," Journal of the American Chemical Society, vol. 38, no. 1, pp. 131-140, 1916.

[41] L. Zhou and Y. Zhang, "Low-valent titanium induced reductive coupling of nitriles with nitro compounds," Synthetic Communications, vol. 28, no. 17, pp. 3249-3262, 1998.

[42] P. Oxley, M. W. Partridge, and W. F. Short, "Amidines. Part VII. Preparation of amidines from cyanides, aluminium chloride, and ammonia or amines," Journal of the Chemical Society (Resumed), pp. 1110-1116, 1947. 


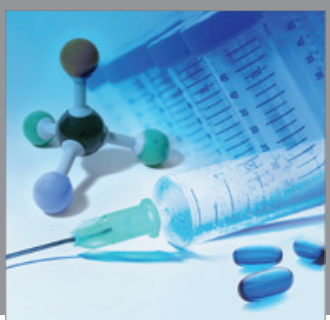

International Journal of

Medicinal Chemistry

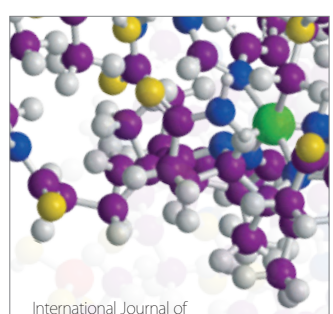

Carbohydrate Chemistry

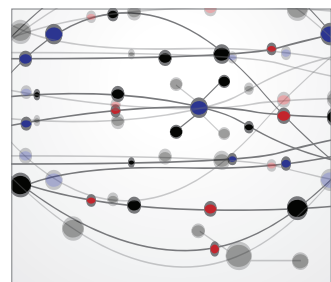

The Scientific World Journal
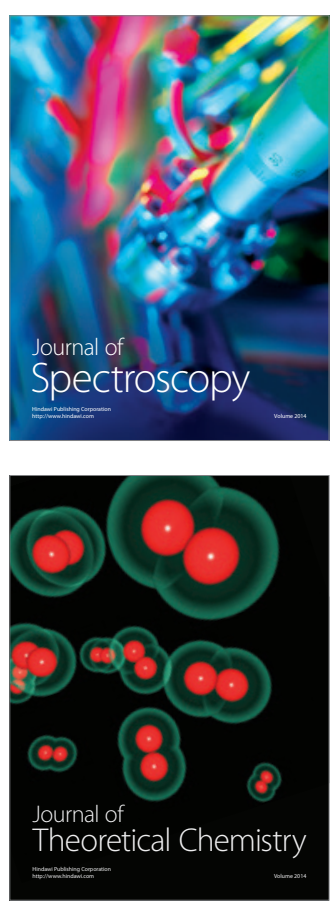
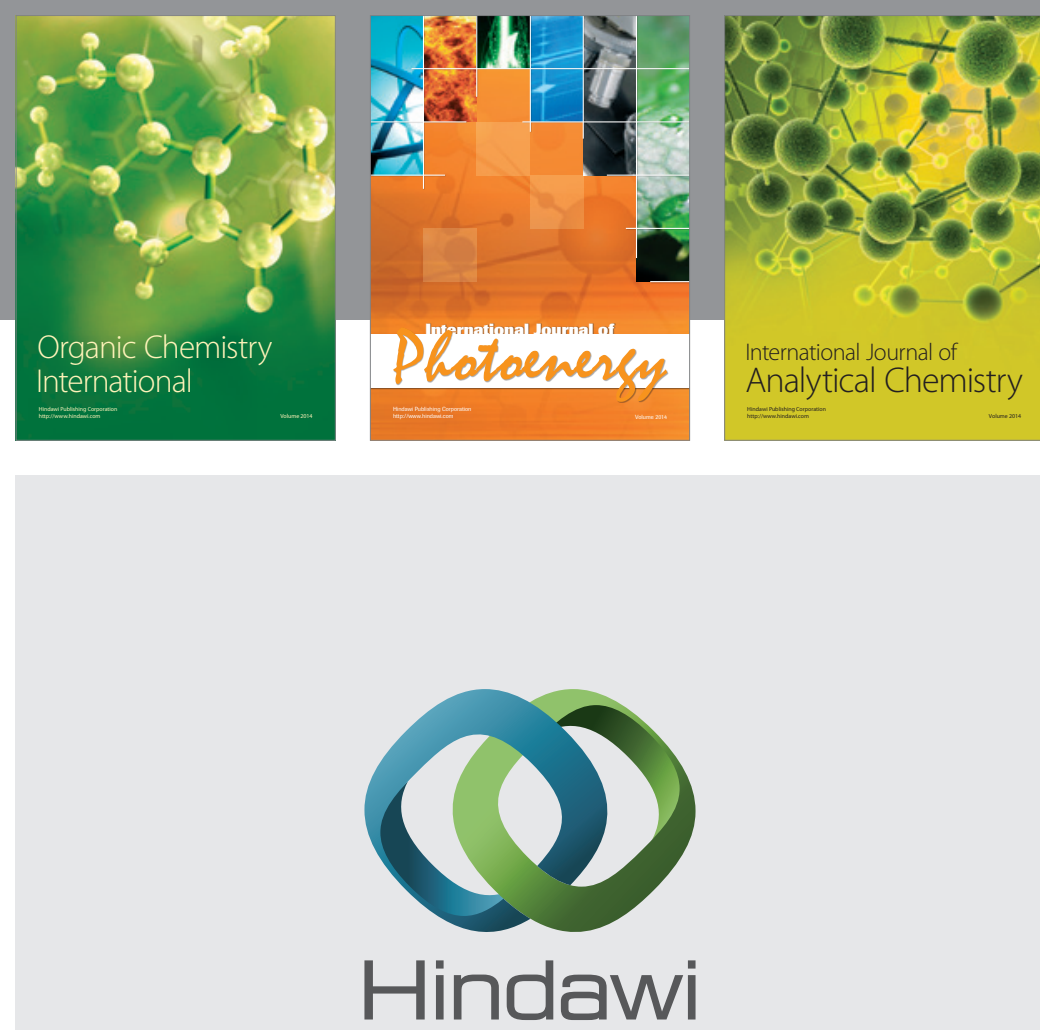

Submit your manuscripts at

http://www.hindawi.com
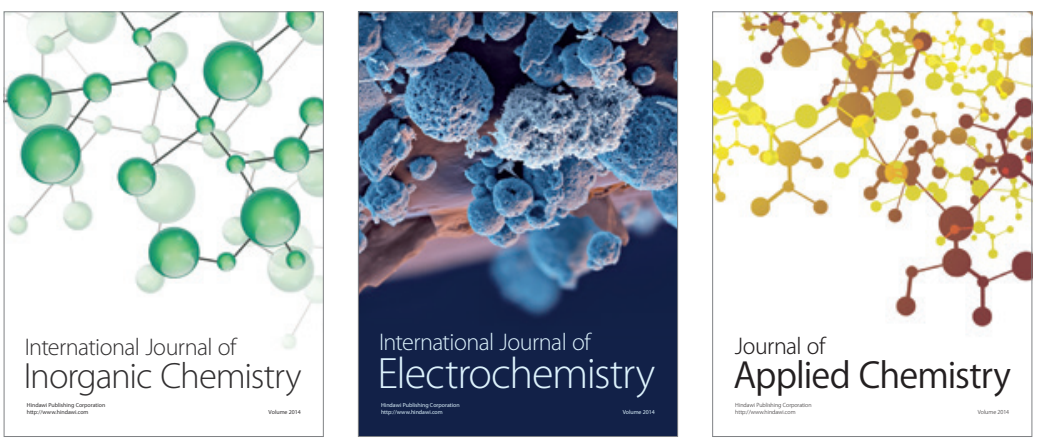

Journal of

Applied Chemistry
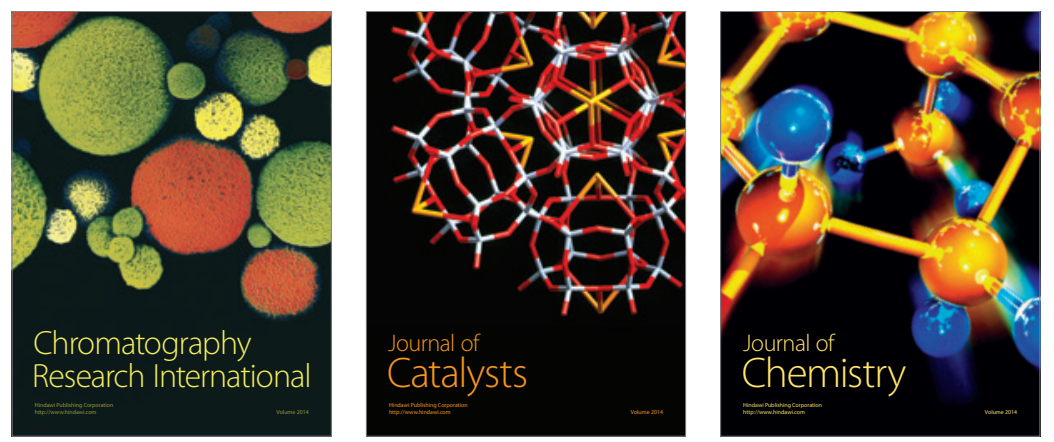
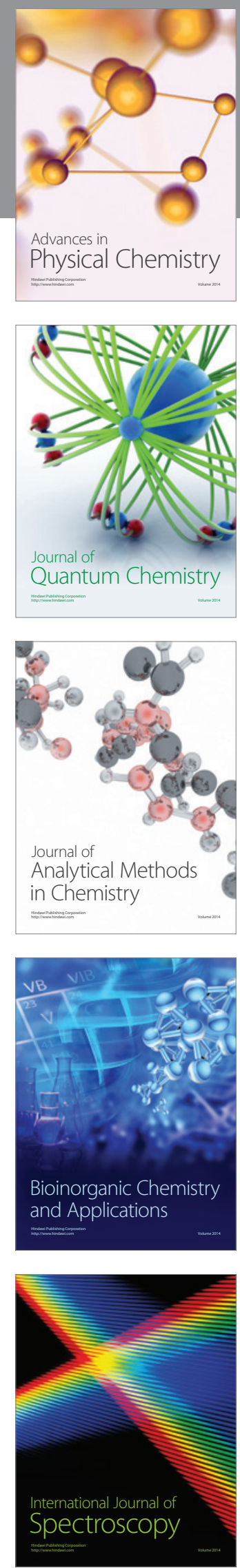